九州大学学術情報リポジトリ

Kyushu University Institutional Repository

\title{
The Subgenus Melandrena of the Genus Andrena of Eastern Asia (Hymenoptera, Andrenidae)
}

$\mathrm{Xu}$, Huan- $\mathrm{li}$

Department of Entomology, College of Agronomy and Biotechnology, China Agricultural University

Tadauchi, Osamu

Laboratory of Entomology, Division of Zoology \& Entomology, Department of Applied Genetics and Pest Management, Faculty of Agriculture, Kyushu University

https://doi.org/10.5109/14046

出版情報 : 九州大学大学院農学研究院紀要. 54 (1)，pp. 109-122，2009-02-27. 九州大学大学院農学研究 院

バージョン :

権利関係 : 


\title{
The Subgenus Melandrena of the Genus Andrena of Eastern Asia (Hymenoptera, Andrenidae)
}

\author{
Xu Huan-lii ${ }^{1 *}$ and Osamu TADAUCHI ${ }^{2}$ \\ ${ }^{2}$ Laboratory of Entomology, Division of Zoology \& Entomology, Department of Applied Genetics \\ and Pest Management, Faculty of Agriculture, Kyushu University, \\ Fukuoka 812-8581, Japan \\ (Received November 2, 2008 and accepted December 5, 2008)0
}

\begin{abstract}
The subgenus Melandrena of the genus Andrena is revised and 17 species are recognized from eastern Asia including one cited species. Four new species, Andrena (Melandrena) vasta, Andrena (Melandrena) tianmushana, Andrena (Melandrena) gilva, Andrena (Melandrena) emeishanica are described and the male of Andrena (Melandrena) sibirica Morawitz is described for the first time. A key to females of East Asian Melandrena is given.
\end{abstract}

\section{INTRODUCTION}

The subgenus Melandrena of the genus Andrena was erected by Peréz in 1890. This large group included 41 Palaearctic species and 29 Nearctic species (Gusenleitner and Schwarz, 2002). Warncke (1968) synonymized the subgenus Gymnandrena Hedicke (1933) and Bythandrena Lanham (1949) with Melandrena. Bouseman and LaBerge (1978) revised North American species of this subgenus. Yasumatsu (1941) listed 75 Andrena described from eastern Asia, in which, 6 species should be allocated in this subgenus. Hirashima (1964) gave a revision and arranged six Japanese species in Gymnandrena. Tadauchi and Hirashima (1987) described a new species Andrena (Melandrena) anzu from Japan. Kim (1970) listed four species of this subgenus distributed in Korea, Kim et al. (1989) gave a revision of Korean species in Gymnandrena. Osytshnjuk (1995) presented a key to Russian Far East of Andrena including seven species of Melandrena. In China, six species belonging to Melandrena were known (Wu, 1965, 1982). In the present study, we examined specimens deposited in the collections of Institute of Zoology, Academia Sinica and the Entomological Laboratory, Kyushu University, Fukuoka and found 16 species, one of which was divided into two subspecies, belonging to Melandrena including four new species, and three species, $A$. (Melandrena) senex Eversmann, A. (Melandrena) koreana Hirashima and A. (Melandrena) sasakii Cockerell, are recorded from China for the first time. We also present a key to female of east Asian Melandrena. The holotypes of new species will be preserved in the Institute of Zoology, Academia Sinica, Beijing and some paratypes will be deposited in the Entomological Laboratory, Kyushu University, Fukuoka.

\section{Subgenus Melandrena Peréz}

Department of Entomology, College of Agronomy and Biotechnology, China Agricultural University, Beijing 100193, China

* Corresponding author (H-l. Xu, E-mail: hanabati@cau.edu.cn)
Melandrena Peréz, 1890, Act. Soc. Linn. Bordeaux, 44: 170; Hedicke, 1933, Mitt. Zool. Mus. Berlin, 19: 216; Lanham, 1949, Univ. California Publ. Ent., 8: 220-221; LaBerge, 1964, Bull. Univ. Nebraska St. Mus., 4: 310-311; Warncke, 1968, Mem. Est. Mus. Zool. Univ. Coimbra, (307): 77-78; Bouseman and LaBerge, 1978, Trans. Amer. Ent. Soc., 104: 276-277. Type species: Apis thoracica Fabricius, 1775, by designation of Michener, 1997.

Gymnandrena Hedicke, 1933, Mitt. Zool. Mus. Berlin, 19: 212; Lanham, 1949, Univ. California Publ. Ent., 8: 221-222; Hirashima, 1957, Mushi, 30: 59-60; LaBerge, 1964, Bull. Univ. Nebraska St. Mus., 4: 311-312; Warncke, 1968, Mem. Est. Mus. Zool. Univ. Coimbra, (307): 77. Type species: Apis thoracica Fabricius, 1775, by original designation.

Cryptandrena Lanham (nec Pittion, 1948): 1949, Univ. California Publ. Ent., 8: 222-223. Type species: Andrena carlini Cockerell, 1901, by original designation.

Bythandrena Lanham, 1950, Ent. News, 61: 140, new name for Cryptandrena Lanham, 1949, autobasic.

Diagnosis: Medium-sized to large bees; facial quadrangle broader than long; vertex tall, facial fovea broad, separated from inner margin of eye by narrow to broad space; clypeus black; labrum sulcate below; subgenal coronet present; malar space evident or linear; genal area broad, rounded; pronotum without humeral angle and ridge; propodeal enclosure small, well defined; propodeal corbicula well developed with long, incomplete anterior fringes; trochanteral floccus imperfect; tibial spurs normal; three submarginal cells present; metasomal terga punctate with hair bands or not. Male with sterna depressed basally, smooth and shiny, without subapical fimbriae; sternum 6 flat.

Key to species of the subgenus Melandrena in eastern Asia (Female)

1. Integument with abundant whitish hairs, especially metasomal terga 2-4 each basal half with long whitish hairs, apical half hyaline with scanty hairs; 
tibial scopal hairs rather short; clypeus densely punctate, unshagreened; process of labrum entire .. senex Eversmann

- Metasomal terga without abundant long hairs

2. Metasomal terga scanty hairs, without tergal hair bands .. 3

- Metasomal terga with hair bands ................... 7

3. Hairs on head and thorax pure brown to black; wing membranes brown; [clypeus coarsely punctate without median impunctate line; process of labrum entire] ........................... vasta $\mathrm{n}$. sp.

- Hairs on head and thorax black mixed with pale; wing membranes dusky .......................... 4

4. Scutellum and posterior part of mesoscutum with black hairs, the reminder of dorsum of thorax with white hairs; genal area, mesepisternum and legs with black hairs; metasomal terga sparsely and minutely punctate, surface metallic blue; pygidial plate small, V-shaped …........... cineraria (L.)

- Dorsum of thorax with fox-red to yellow hairs, without black hairs; metasomal terga distinctly punctate, surface black ......................... 5

5. Process of labrum narrowed toward apex, entire; clypeus coarsely punctate, less roughened; flagellar segment 1 as long as flagellar segment 2 plus 3; pronotum notched at subapical margin; clypeus with yellow hairs .............. parathoracica Hirashima

- Process of labrum broad, entire or deeply emarginate; clypeus coarsely punctate, strongly roughened; flagellar segment 1 shorter or longer than segment 2 plus 3; pronotum entire at subapical margin; face of head with brown hairs ........................ 6

6. Process of labrum emarginate; clypeus without median impunctate line or indistinct apically; flagellar segment 1 longer than segment 2 plus 3 thoracica sinensis Cockerell

- Process of labrum entire; clypeus with tessellate longitudinal space; flagellar segment 1 shorter than flagellar segment 2 plus 3 ... crassepunctata Cockerell

7. Pronotum notched at subapical margin, with short, longitudinal line ............................. 8

- Pronotum entire, without median longitudinal line

Thoracic dorsum mainly with brown hairs; clypeus convex, coarsely punctate with median shiny impunctate space; propodeal enclosure coarsely rugosed mostly; posterior depressions of metasomal terga broad, weakly punctate ... watasei Cockerell

- Thoracic dorsum with yellow to fulvous hairs, if admixed black hairs, then clypeus smooth and shiny with indistinct median impunctate line; clypeus coarsely punctate without median impunctate space ..................................... 9

9. Hairs on head brown; process of labrum broad, deeply emarginate, metasomal terga with indistinct hair bands .................... tianmushana n. sp.

- Hairs on head yellow to dull white except on vertex; process of labrum entire or emarginate; metasomal terga with distinct hair bands
10. Clypeus coarsely rugulate with sparse punctures; process of labrum deeply emarginate; propodeal enclosure coarsely rugulate with depressed suture medially ........... sibirica sapporensis Hirashima

- Clypeus coarsely punctate, weakly shiny; process of labrum entire, weakly emarginated in occasion; propodeal enclosure weakly rugulate basally ..... 11

11. Metasomal terga densely punctate with moderate punctures; pygidial plate U-shaped; length 13-14 mm ................................. koreana Hirashima

- Metasomal terga densely punctate with small punctures; pygidial plate V-shaped; length 11-12.5 mm

12

12. Clypeus strongly convex, shiny; process of labrum trapezoidal; apical areas of metasomal terga sparsely and weakly punctate ........ edashigei Hirashima

- Clypeus weakly convex, tessellate; process of labrum narrowed at apex; metasomal terga evenly densely punctate ....................... gilva n. sp.

13. Flagellar segment 1 shorter than segment 2 plus 3 in length; clypeus sparsely and weakly punctate with median impunctate space, surface weakly tessellate; tibial scopal hairs short; metasomal terga 2-4 evenly and densely punctate with small punctures; apical fasciae weak ............ anzu Tadauchi et Hirashima

- Flagellar segment 1 as long as or longer than flagellar segment 2 plus 3 in length; clypeus coarsely punctate, surface roughened .................... 14

14. Metasomal terga 2-4 with broad white hair patches laterally; process of labrum emarginate; thorax with dull white hairs except short and narrow black hairs on posterior margin of mesoscutum and anterior part of scutellum; metasomal terga smooth and shiny, weakly punctate ........... comta Eversmann

- Metasomal terga with short white hair bands; process of labrum entire or emarginate; metasomal terga tessellate ................................. 15

15. Flagellar segment 1 longer than segment 2 plus 3 in length; [process of labrum entire; propodeal enclosure strongly rugulate mostly; metasomal terga 2-4 evenly and densely punctate with small punctures] .................... emeishanica n. sp.

- Flagellar segment 1 as long as flagellar segment 2 plus 3 in length ................................. 16

16. Process of labrum small, emarginate; mesoscutum coarsely punctate; propodeal enclosure rugulate at basal half with median suture; metasomal terga densely punctate with moderate punctures .......................... sibirica sibirica Morawitz

- Process of labrum large, entire; mesoscutum sparsely and minutely punctate; propodeal enclosure weakly rugulate basally; metasomal terga sparsely punctate with minute punctures ............. sasakii Cockerell

\section{Andrena (Melandrena) senex Eversmann}

(Fig. 1: A-E)

Andrena senex Eversmann, 1852, Bull. Soc. Nat. Moscou, 25 (2): 27 [female, M-Asia]; Warncke, 1967, Eos, 43: 307 [in list].

Andrena ovinella Friese, 1914, Stett. ent. Ztg., 75: 
224-225 [female and male, Mongolia]; Yasumatsu, 1941, Peking nat. Hist. Bull., 15: 279 [in list]; Warncke, 1967, Eos, 43: 296 [synonymy].

Andrena (Melandrena) senex: Gusenleitner and

Schwarz, Entomofauna, Suppl., 12: 687-688.

Redescription.

Female. BL 11.2-14.5 mm, WL 10.2-11.7 mm ( $\mathrm{n}=10)$.

Color: Flagellum reddish brown beneath; mandible with apical third or more reddened; wing membranes weakly brown, veins and pterostigma yellowish brown; tibial spurs testaceous; posterior depressions of metasomal terga yellowish translucent.

Pubescence: Hairs on head rather dense, dull white except face along inner margin of eye, vertex and genal area outer margin of eye mixed with brown; those on clypeus 400-500 $\mu$; those on vertex about $700 \mu$; facial fovea dark brown. Hairs on thorax rather dense, pale white to white; those on mesoscutum and scutellum 500-900 $\mu$; those on mesepisternum white; propodeal corbicula well developed with incomplete anterior fringes, with internal long, simple hairs; trochanteral floccus imperfect, short at base, silver white; femoral floccus dense; tibial scopal hairs short, compact, simple, bright brown; basitarsi with brown hairs. Hairs on metasomal terga 1-5 long, dense, suberect, composed of basal white fasciae, apical margins without hair bands; caudal fimbria bright brown; sterna 2-5 with long, sparse subapical fimbriae, white to bright brown.

Structure: Head: HL/HW = 0.73. HW: MsW: MtW = 3.9: 3.9: 4.4. Vertex dulled by minute PP above lateral ocelli, weakly tessellate on top of eye. OOD: OCD: POD $=0.8: 0.5: 0.3$. FL1= FL2+3, FL2 slightly shorter than FL3, FL2 broader than long, intermediate segments about as broad as long. Eyes with inner margins paralleled. Facial fovea exceeding below a line at lower margin of antennal fossae, close to eye, FVL $=1.7 \mathrm{~mm}, \mathrm{FVW}$
$=0.5 \mathrm{~mm}$. Supraclypeal area dulled by minute PP. Face above antennal fossae with fine longitudinal rugulae and coarse interrugal PP, surface dulling. Facial quadrangle broader than long (about 2.9: 2.2). Clypeus nearly flattened, surface weakly punctate-tessellate medioapically, sparse small PP, IS $<1$ basally, without median impunctate line, $\mathrm{CPL}=1.2 \mathrm{~mm}$. Process of labrum broad at base, narrowed toward apex, rounded or entire apically, surface smooth and shiny. Labrum apical to process transversely sulcate, with several short cristae. Lower paraocular area weakly tessellate and minutely punctate. Malar space linear. Genal area broader than eye, GW: EW = 1.0:0.8, surface weakly tessellate, shiny with obscure PP posteriorly. Mesosoma: Pronotum weakly tessellate with dense minute PP. Mesoscutum densely tessellate, opaque, surface with PPø15 $\mu$, IS = 1-2. Scutellum weakly tessellate medially, scattered minute PP, irregular in distribution, densely punctate marginally. Propodeal enclosure densely tessellate, with basal strong, short rugulae; dorsal face of propodeum densely tessellate with sparse minute PP. Mesepisternum dulled by coarse small PP, surface weakly tessellate. Vein 1st $m-c u$ meeting second submarginal cell at middle of cell. Metasoma: metasomal terga smooth to weakly tessellate, broadly shiny; tergum 1 with sparse minute PP, IS = 2-4 medially, PP denser near apical area; terga 2-4 with basal areas with close fine PP, IS = 1-2, subhyaline apical areas with weak to obscure minute PP; posterior depressions or terga well indicated; pygidial plate $\mathrm{V}$-shaped with rounded apex, with internal punctate raised area. Sterna 2-5 impunctate basally, finely punctate apically with PP, IS = 1, surface broadly shiny.

Male. BL 8.0-10.0 mm, WL 7.5-8.7 mm ( $\mathrm{n}=5)$.

Color: Flagellum reddish brown beneath; mandible reddened apically; wing membranes hyaline to pale

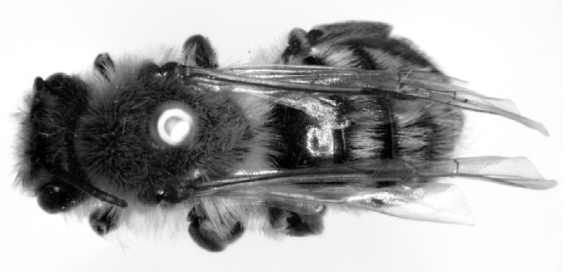

A

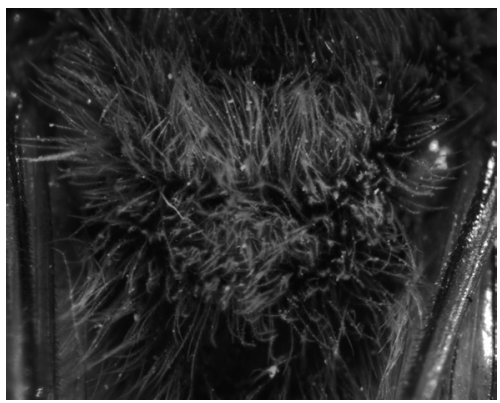

D

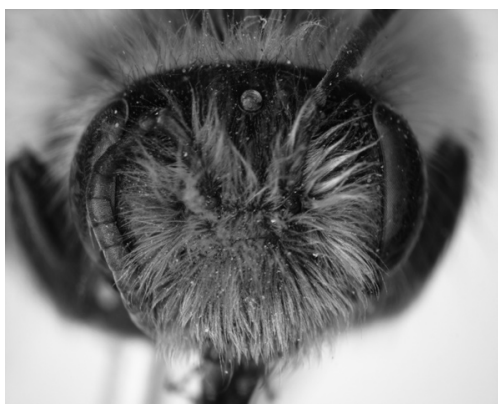

B

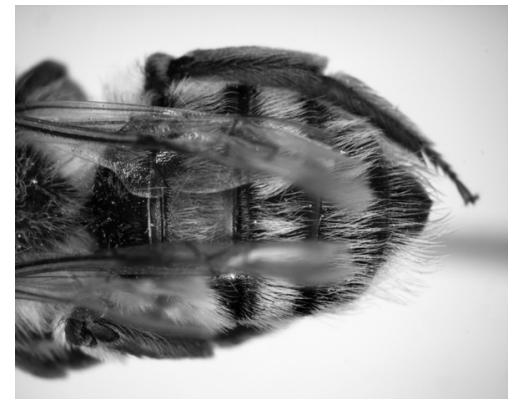

E

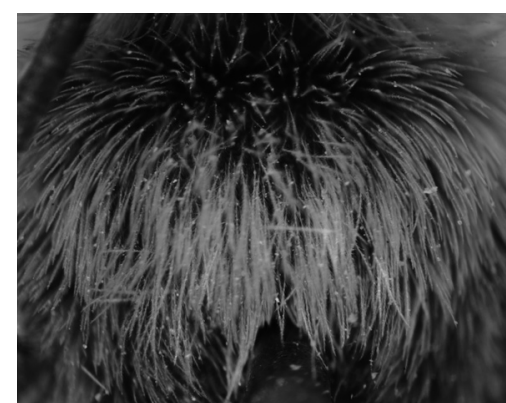

C

Fig. 1. A-E. Andrena (Melandrena) senex Eversmann, female. A: general habitus in dorsal view; B: head in frontal view; $\mathrm{C}$ : mesoscutum; D: propodeum; E: metasomal terga. 
brown, veins and pterostigma reddish yellow; tibial spurs yellowish; posterior depressions of metasomal terga yellowish translucent.

Pubescence: Hairs on head as in female except vertex with few brown hairs; those on clypeus rather long $(900 \mu)$, dense, white; those on vertex 600-900 $\mu$, white. Hairs on mesoscutum and scutellum 600-700 $\mu$, pale white; those on mesepisternum longer, whitish. Hairs on metasomal terga similar in female, but short and sparse at basal areas; sterna 2-5 scattered rather sparse white hairs, without subapical fimbriae.

Structure: Head: HL/HW = 0.7. HW: MsW: MtW = 3.2: 2.7: 3.2. Vertex flat, densely tessellate. OOD: POD: $\mathrm{OCD}=0.7:$ 0.5: 0.3. FL1 > FL3, FL2 < FL3, FL2 about quadrate, following segments longer than broad. Eyes with inner margins slightly diverging vertex. Supraclypeal area and face above antennal fossae sculptured as in female. Facial quadrangle broader than long (about 2.2:1.7). Clypeus sculptured as in female, but PP smaller, $\mathrm{CPL}=0.7 \mathrm{~mm}$. Process of labrum as in female, smaller. Mandibles long, decussate. Lower paraocular area finely tessellate and punctate. Malar space linear. Genal area broader than eye, GW: EW = 1.0: 0.76 , surface densely tessellate with weak longitudinal rugulae posteriorly. Mesosoma: Pronotum finely tessellate with obscure PP. Mesoscutum and scutellum densely tessellate, opaque, PP obscured. Propodeal enclosure as in female; dorsal face of propodeum densely granulate with weak PP, surface shagreening. Mesepisternum densely tessellate, dulled by minute PP. Vein 1st $m-c u$ meeting second submarginal cell beyond middle of cell. Metasoma: Metasomal terga weakly tes- sellate, shiny; tergum 1 scattered obscure microscopic PP; terga 2-4 with sparse and weak minute PP at basal areas, IS = 1-3; posterior depressions of terga as in female in form and sculpture. Sterna smooth and shiny, scattered obscure PP at basal areas; sternum 6 weakly reflected apically, not emarginated.

Specimens examined. Syntypes of A. ovinella (Berlin Zoological Museum), 2 females and 1 male, Weiske leg. Other material: [CHINA] Heilongjiang Province: Harbin: 1 female, 16. vii. 1947; 1 female, 26. iv. 1952; 1 female and 1 male, 24. iv. 1953; 1 female, 7. v. 1954. Jilin Province: Kaiyen (now is Kaiyuan): 2 females, 22. v. 1936 (R. Kimishima); 2 females, 12-13. vi. 1937 (R. Kimishima). Gansu Province: Wuwei city: 2 females and 4 males (O. Tadauchi); 2 males (H-l. Xu), 29. iv. 1996.

Distribution. China (new record, Gansu, Heilongjiang, Jilin Provs.); Mongolia.

Floral association. Salix sp.

\section{Andrena (Melandrena) vasta n. sp.}

(Fig. 2: A-E)

Female. BL $15.0 \mathrm{~mm}$, WL $12.0 \mathrm{~mm}(\mathrm{n}=1)$.

Color: Flagellum dark reddish brown beneath except at base; mandible with apical half more reddened; wing membranes infumate; veins and pterostigma reddish brown; tibial spurs reddish brown; posterior depressions of metasomal terga black.

Pubescence: Hairs on body brown to black; those on clypeus scanty broadly, brown hairs (200-400 $\mu$ ) at base and lateral area; those on antennal area black;

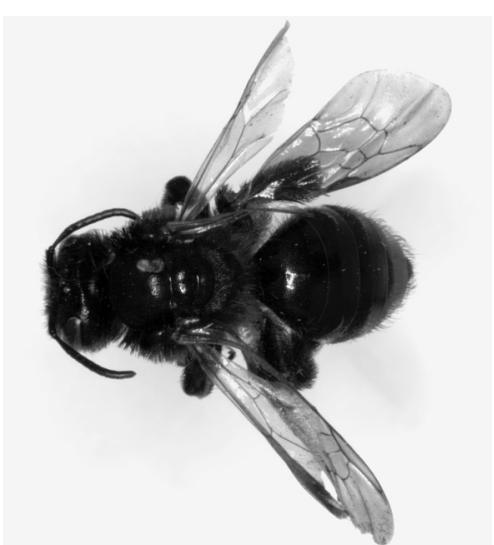

A

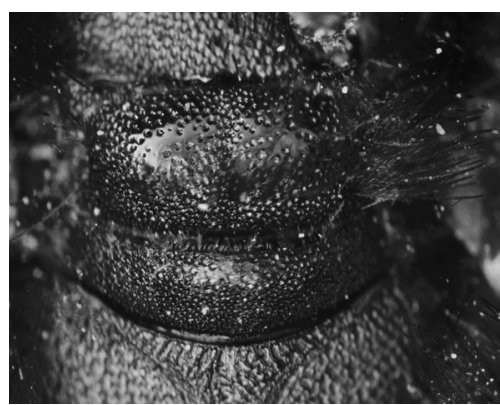

D

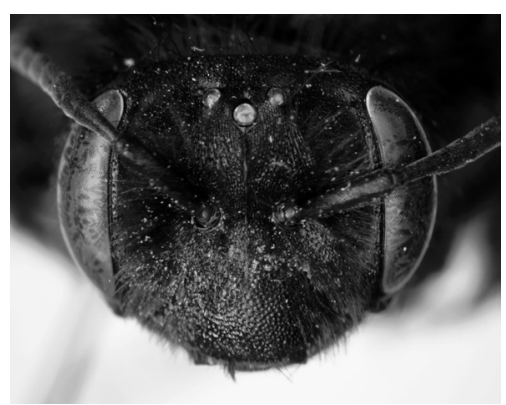

B

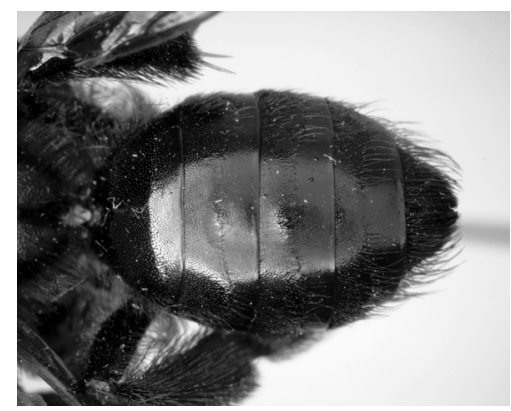

$\mathbf{E}$

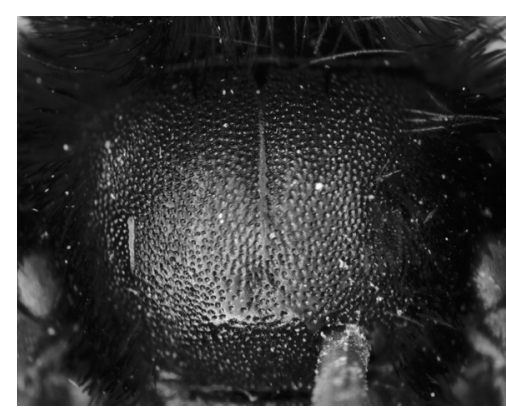

C

Fig. 2. A-E. Andrena (Melandrena) vasta $\mathrm{n}$. sp., female. A: general habitus in dorsal view; B: head in frontal view; C: mesoscutum; D: propodeum; E: metasomal terga. 
those on vertex 400-600 $\mu$, black; facial fovea brown. Hairs on mesoscutum 500-600 $\mu$, dense, black; those on scutellum long and dense, black; those on mesepisternum about $700 \mu$, black above, brown below; propodeal corbicula developed with incomplete anterior fringes, black, with internal sparse, simple hairs; trochanteral floccus imperfect, short, brown; femoral floccus dense; tibial scopal hairs moderately long, dense, black. Hairs on metasomal terga scanty; terga 1-3 with short and sparse black hairs at lateral margins; tergum 4 with sparse black hairs medially; caudal fimbria black; sterna 2-5 with sparse, incomplete brown subapical fimbriae.

Structure: Head: HL/HW = 0.83. HW: MsW: MtW = 4.2: 4.3: 4.8. Vertex finely tessellate, feebly shiny, dulled by coarse PP above lateral ocelli. OOD: POD: OCD = 0.85: 0.5: 0.45. FL1 > FL2+3, FL2 < FL3 broader than long, intermediate segments about as long as broad. Eyes with inner margins subparalleled. Facial fovea broad, separated from eye by narrow punctate space, exceeding below a line at lower margin of antennal fossae, FVL $=1.8 \mathrm{~mm}, \mathrm{FVW}=0.6 \mathrm{~mm}$. Supraclypeal area roughened by coarse minute PP. Face above antennal fossae with weak longitudinal rugulae and weak interrugal PP, surface dulling. Facial quadrangle broader than long (about 3.1: 2.7). Clypeus well convex, surface coarsely reticularly punctate with $\mathrm{PP} \varnothing 30 \mu$. without median impunctate line, $\mathrm{CPL}=1.3 \mathrm{~mm}$. Process of labrum broad, trapezoidal, entire apically. Labrum apical to process weakly sulcate transversely, without crista. Lower paraocular area coarsely punctate with РРø20 $\mu$. Malar space short, about one-fourth as long as width of basal mandible. Genal area broader than eye, GW: $\mathrm{EW}=1.1$ : 0.9, surface finely tessellate, feebly shiny with close minute PP near eye. Mesosoma: Pronotum reticularly shagreened with obscure minute PP. Mesoscutum densely tessellate, dulled by crowded PPø20-40 $\mu$, IS $<0.5$. Scutellum crowdedly punctate with PPø20-30 $\mu$, shiny medially. Propodeal enclosure triangular, narrow, weakly wrinkled basally, densely tessellate apically; dorsal face of propodeum shagreened by coarse PP. Mesepisternum shagreened. Hind tibiae cuneate. Vein 1st $m-c u$ meeting second submarginal cell at middle of cell. Metasoma: Metasomal terga weakly tessellate, shiny; terga 1-3 with distinct PРø15$20 \mu$, IS $=0.5-1$ at basal areas, weak and minute PP at apical areas; tergum 4 with sparser PP, apical with obscure PP; posterior depressions of terga broad, occupied one half of segmental length on terga 2-3, twothirds on tergum 4, moderately indicated; pygidial plate V-shaped, with internal weak raised area. Sterna 2-5 weakly tessellate, narrowly impunctate basally, broadly and coarsely punctate apically.

Male. Unknown.

Type material. Holotype female, Fuyun, 1,200 m, Xinjiang Uygur Autn. Region, China, 12. vii. 1960 (S-y. Wang).

Remarks. This species is described based on one female. It is similar to European Andrena morio Brullé in having the black hairs on integument. It differs from morio in the broad and entire labral process, the blackish tibial scopal hairs, the less rugulosed propodeal enclosure and the strongly punctate metasomal terga.

Distribution. China (Xinjiang Uygur Autn. Region). Floral association. Not available.

\section{Andrena (Melandrena) cineraria (L.)}

Apis cineraria Linnaeus, 1758, Syst. nat. (Ed., 10) 1: 575 [Europe].

Andrena cineraria: Morawitz, 1880, Biol. Bull. Acad. Sci., St. Petersbourg, 10: 3-4: 483 [in list, Mongolia]. Alfken, 1936, Ark. Zool., 27A(37): 3; Yasumatsu, 1941, Peking nat. Hist. Bull., 15: 274; Yasumatsu, 1946, Mushi, 17: 20 [in list, China].

Andrena (Gymnandrena) cineraria: Hirashima: 1957, Mushi, 30: 62; Wu, 1982, Insects Xizang, Vol. 2, Apoidea, 384 [in list].

Andrena (Melandrena) cineraria: Warncke, 1968, Mem. Est. Mus. Zool. Univ. Coimbra, (307): 78 [in list]; Osytshnjuk, 1995, Key Insects Russian Far East, 4 (1): 504, 523 [female and male, in key]; Gusenleitner and Schwarz, Entomofauna, Suppl., 12: $178-179$.

Other synonymies: See Gusenleitner and Schwarz (2002).

Specimens examined. [CHINA] Gansu Province: 3 females, Yongchang County, 11. viii. 1996 (O. Tadauchi and $\mathrm{H}-\mathrm{l}$. Xu); 1 female, Daban, Lingtao County, 17. viii. 1996 (O. Tadauchi); 1 female, Lanzhou city, 18. viii. 1996 (O. Tadauchi). Qinghai Province: 1 female, Jiayi, Qinghai Lake, 15. viii. 1995 (O. Tadauchi). [MONGOLIA] Central aimak: 2 females, 7-11. vi. 1966 (Z. Kaszab). Ostgobi aimak: 1 female and 1 male, 21. vi. 1963 (Z. Kaszab).

Distribution. China (Xizang, Qinghai, Xinjiang, Gansu, Inner Mongolia); Mongolia; Russia (Far East, European Russia); Europe; North Africa; Asia Minor; Turkmenistan; Iran; Pakistan.

Floral associations. China: Brassica campestris, Allium tuberosium, Astragalus sinicus.

\section{Andrena (Melandrena) thoracica sinensis Cockerell}

Andrena sinensis Cockerell, 1910, Trans. Amer. ent. Soc., 36: 249 [female, China]; Cockerell, 1931, Amer. Mus. Nov., 466: 11 [female in key].

Andrena thoracica var. sinensis: Alfken, 1936, Ark. Zool., 27A(37): 3 [Kansu, China]. Yasumatsu, 1941, Peking nat. Hist. Bull., 15: 281 [in list].

Andrena thoracica: Radoszkowski, 1890, Horae Soc. ent. Ross., 24: 230 [Korea]; Sickmann, 1895, Zool. Jahrb., 8: 235 [China]; Cockerell, 1911, Proc. U. S. nat. Mus., 40: 244 [China]; Cockerell, 1931, Amer. Mus. Nov., 466: 11 [female, in key]; Wu, 1965, Econ. Insects Faun. China IX, Apoidea, 32.

Andrena (Melandrena) thoracica: Osytshnjuk, 1995, Key Insects Russian Far East, 4 (1): 504, 523 [female and male, in key]; Gusenleitner and Schwarz, Entomofauna, Suppl., 12: 759-760. 
Other synonymies: See Gusenleitner and Schwarz (2002).

Specimens examined. [CHINA] Gansu Province: A series of specimens collected by O. Tadauchi and H-l. Xu: 38 females and 9 males, Wuwei, 28-30. iv. 1995; 10 females and 6 males, Zhangye, 1. v. 1996; 50 females, Jiuquan, 5. v. 1996; 3 females, Mingle, 12. viii. 1996. Beijing: 3 females, 12. v. 1981 (Y-r. Wu). Shandong: 1 female, Yantai, 30. vi. 1973 (S-d. Liu).

Distribution. China (Gansu, Beijing, Shandong Provs.); Korea; Russia (Far East area).

Floral association. Brassica campestris.

\section{Andrena (Melandrena) parathoracica Hirashima}

Andrena (Gymnandrena) parathoracica Hirashima, 1957, Mushi, 30: 63 [female and male, Japan]; Hirashima, 1964, J. Fac. Agr., Kyushu Univ., 13: 59-62; Hirashima, 1966, J. Fac. Agr., Kyushu Univ., 14: 106, 119 [female and male, in key]; Kim, 1970, Ill. Enc. Faun. and Flor. Korea, 11: 660 [Korea]; Kim et al., 1990, Ent. Res. Bull., 16: 4.

Andrena (Melandrena) parathoracica: Osytshnjuk, 1995, Key Insects Russian Far East, 4 (1): 504, 523 [female and male, in key]; Gusenleitner and Schwarz, Entomofauna, Suppl., 12: 579.

Specimens examined. Holotype male and allotype female (Kyushu Univ.).

Distribution. Japan (Hokkaido, Honshu, Shikoku, Kyushu, Tsushima); Korea (southern and central Korea); Russia (Far East area).

Floral association. Not available.

\section{Andrena (Melandrena) crassepunctata Cockerell}

Andrena crassepunctata Cockerell, 1931, Amer. Mus. Nov., (446): 10-11 [female, China]; Yasumatsu, 1941, Peking nat. Hist. Bull., 15: 275 [in list]; Kim, 1970, Ill. Encycl. Faun. and Flor. Korea, 11: 659-660 [Korea].

Andrena crassipunctata (sic): Wu, Econ. Insects Faun. China XI, Apoidea, 32 [China].

Andrena (Gymnandrena) crassepunctata: Hirashima, 1957, Mushi, 30: 63.

Andrena (Gymnandrena) crasspunctata (sic): Kim et al., 1990, Ent. Res. Bull., 16: 2-3 [Korea].

Andrena (Melandrena) crassepunctata: Tadauchi et al., 1997, Esakia, (37): 191 [Korea]; Gusenleitner and Schwarz, Entomofauna, Suppl., 12: 203-204.

Specimens examined. Holotype female (American Museum of Natural History, New York), Zo-ze, Jiangsu, China, 17. iv. 1930 (O. Piel). Other material: [CHINA] Beijing: 1 female, Miaofengshan, 15. v. 1996 (O. Tadauchi). Liaoning: 2 females, Mt. Daiosho, 19. v.- 9. vi. 1935 (M. Hanano); 1 female, Fengtian, Shenyang, 17. v. 1936 (R. Kimishima). [KOREA] 1 female, Suigen, 19. iv. 1924 (K. Sato).

Distribution. China (Beijing, Liaoning Prov.); Korea (southern Korea).

Floral association. Not available.

\section{Andrena (Melandrena) watasei Cockerell}

Andrena watasei Cockerell, 1913, Ann. Mag. nat. Hist., (8)11: 187 [female and male, Japan]; Yasumatsu, 1941, Peking nat. Hist. Bull., 15: 281 [in list]; Uéda, 1954, Trans. Shikoku ent. Soc., 4: 50 [in list].

Andrena (Gymnandrena) watasei: Hirashima, 1957, Mushi, 30: 66; Hirashima, 1964, J. Fac. Agr., Kyushu Univ., 13: 55-57; Hirashima, 1966, J. Fac. Agr., Kyushu Univ., 14: 107, 119 [female and male, in key].

Andrena (Melandrena) watasei: Osytshnjuk, 1995, Key Insects Russian Far East, 4 (1): 504, 524 [female and male, in key]; Gusenleitner and Schwarz, Entomofauna, Suppl., 12: 828-829.

Andrena azarearum Cockerell, 1930, Ann. Mag. nat. Hist., (10)5: 115 [female, Japan].

Specimens examined. A series of specimens preserved in Kyushu University are examined.

Distribution. Japan (Hokkaido, Honshu, Sado Is., Shikoku, Kyushu, Tsushima Is., Yakushima Is.); Russia (Far East area).

Floral association. Brassica campestris.

\section{Andrena (Melandrena) tianmushana n. sp.}

(Fig. 3: A-E, Fig. 4: A-E)

Female. BL 12.0-13.5 mm, WL 10.0-11.7 mm ( $\mathrm{n}=14)$.

Color: Flagellum reddish brown beneath; mandible with apical half or more reddened; wing membranes dusky, veins and pterostigma reddish brown; tibial spurs testaceous; posterior margins of metasomal terga yellowish brown translucent.

Pubescence: Hairs on head sparse, brown; those on clypeus $200-500 \mu$; those on vertex about $500 \mu$; facial fovea dark brown. Hairs on thorax rather dense, yellow; those on mesoscutum and scutellum 300-500 $\mu$; those on mesepisternum paler; propodeal corbicula well developed with incomplete anterior fringes, with internal long, sparse, simple hairs; trochanteral floccus nearly perfect, dull white; femoral floccus dense; tibial scopal hairs long, simple, bright brown; basitarsi with brown hairs. Hairs on metasomal terga scanty; terga 2-4 with broadly interrupted white hair bands; caudal fimbria bright brown; sterna 2-5 with long, sparse subapical fimbriae, brown.

Structure: Head: HL/HW = 0.81. HW: MsW: MtW = 3.7: 3.7: 4.2. Vertex dulled by minute PP above lateral ocelli, weakly tessellate on top of eye. OOD: OCD: POD $=0.8: 0.4: 0.3$. FL1 $=$ FL2 +3, FL2 = FL3 as broad as long, intermediate segments longer than broad. Eyes with inner margins paralleled. Facial fovea exceeding below a line at lower margin of antennal fossae, separated from eye by a narrow space, $\mathrm{FVL}=1.7 \mathrm{~mm}, \mathrm{FVW}=0.6 \mathrm{~mm}$. Supraclypeal area shagreened by minute PP. Face above antennal fossae with fine longitudinal rugulae and weak interrugal PP, unshagreened. Facial quadrangle broader than long (about 2.6: 2.4). Clypeus well convex, surface ruguso-punctate with coarse PРø40 $\mu$, 


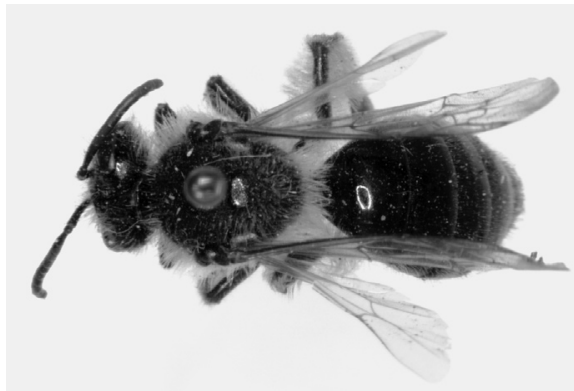

A

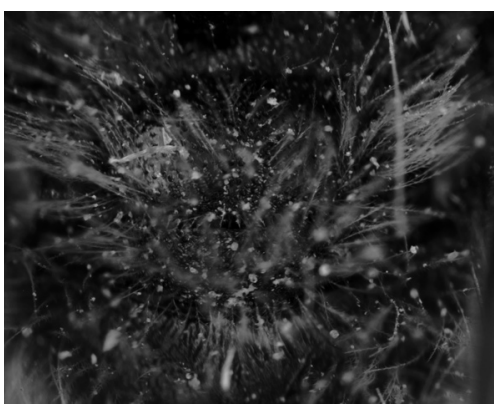

D

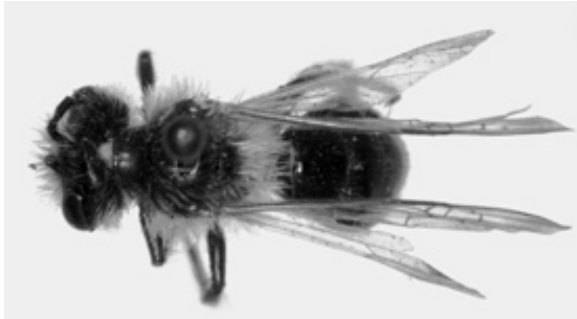

$\mathbf{A}$

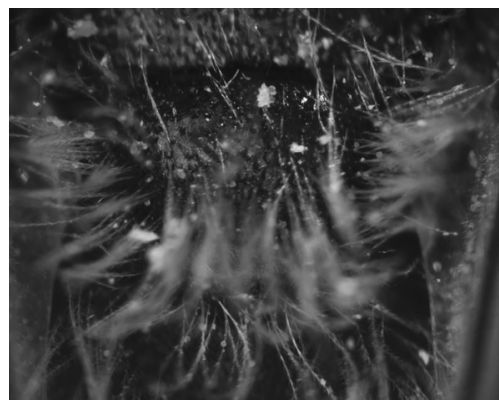

D

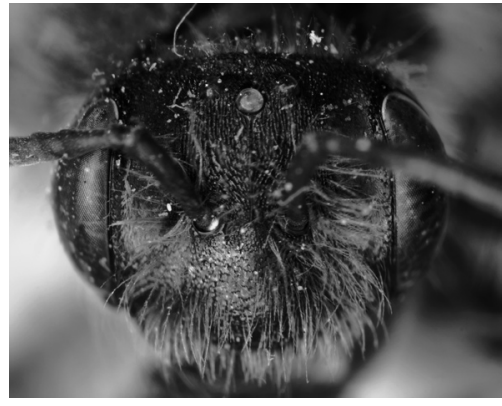

B

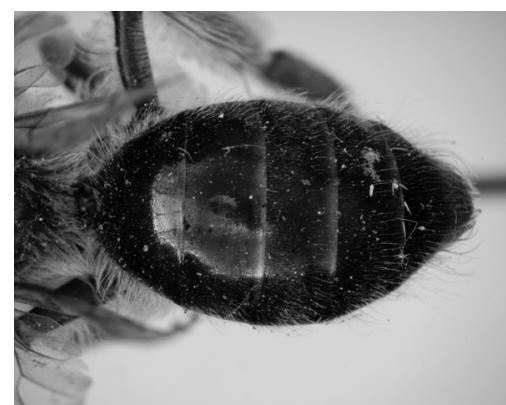

$\mathbf{E}$

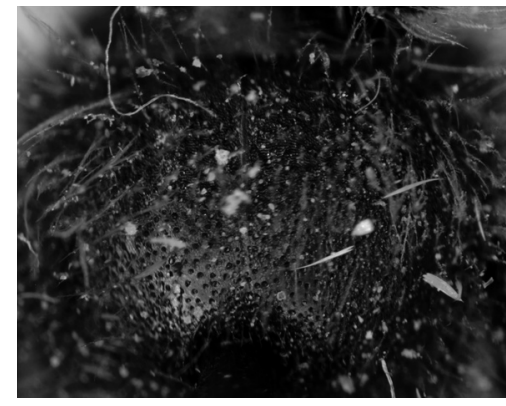

C

Fig. 3. A-E. Andrena (Melandrena) tianmushana n. sp., female. A: general habitus in dorsal view; B: head in frontal view; C: mesoscutum; D: propodeum; E: metasomal terga.

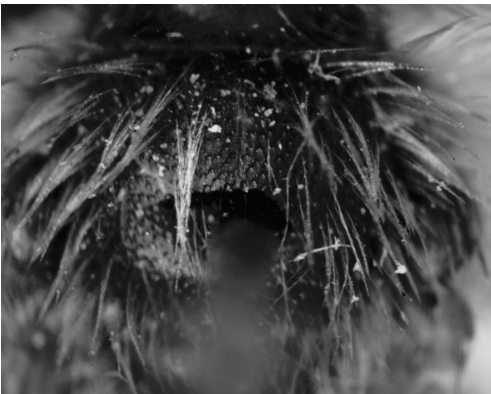

C

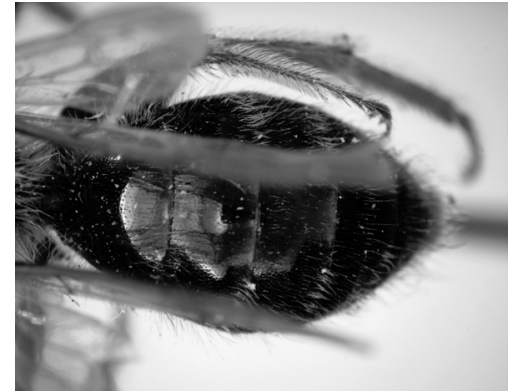

$\mathbf{E}$ surface roughened except basally; without median impunctate line, $\mathrm{CPL}=1.2 \mathrm{~mm}$. Process of labrum broad, trapezoidal, emarginate apically, surface smooth and shiny. Labrum apical to process transversely sulcate, with median cristae. Lower paraocular area weakly tessellate with obscure PP. Malar space nearly linear. Genal area broader than eye, GW: $\mathrm{EW}=1.0$ : 0.9, surface weakly tessellate, shiny with sparse, obscure minute PP posteriorly. Mesosoma: Pronotum weakly tessellate with sparse minute PP, IS = 2, subapical margin notched medially with short longitudinal line. Mesoscutum and scutellum crowdedly punctate with PPø20 $\mu$, IS $<0.5$. Propodeal enclosure densely tessellate laterally and apically, finely rugosed basally; dorsal face of propodeum densely tessellate, weakly roughened by punctation. Mesepisternum with sparse tessellate-punctation. Vein 1 st $m-c u$ meeting second submarginal cell slightly beyond middle of cell. Metasoma: metasomal terga smooth to weakly tessellate, shiny; tergum 1 with PPø15-20 $\mu$, IS $=0.5-1$; terga $2-4$ with close PPø15 $\mu$, IS 
$<0.5$, PP weaker at apical area; posterior depressions or terga well indicated; pygidial plate U-shaped, internal surface densely punctate, without triangular area. Sterna 2-5 narrowly impunctate basally, finely punctate apically with PP, IS = 1-1.5.

Male. BL 9.5-10.0 mm, WL $8.0 \mathrm{~mm}(\mathrm{n}=2)$.

Color: Flagellum dark reddish brown beneath; mandible reddened apically; wing membranes and veins as in female; tibial spurs yellowish; posterior depressions of metasomal terga reddish brown.

Pubescence: Hairs on clypeus $600 \mu$, rather dense, yellowish white; those on vertex 500-550 $\mu$, sparse, fulvous. Hairs on mesoscutum and scutellum 500-700 $\mu$, yellow; those on mesepisternum yellowish. Hairs on metasomal terga short, sparse, those on terga 1-2 pale white; those on terga 3-6 brown; sterna 2-5 scattered rather sparse white hairs, without subapical fimbriae.

Structure: Head: HL/HW = 0.74. HW: MsW: MtW = 3.2: 2.7: 2.9. Vertex finely tessellate. OOD: POD: OCD $=0.7: 0.4: 0.3$. FL1 = FL2 longer than broad, following segments longer than broad. Eyes with inner margins paralleled. Supraclypeal area and face above antennal fossae sculptured as in female. Facial quadrangle broader than long (about 2.2: 2.0). Clypeus as in female in form and sculpture, but PP smaller, $\mathrm{CPL}=0.9 \mathrm{~mm}$. Process of labrum as in female. Mandibles long, decussate. Lower paraocular area weakly tessellate and punctate. Malar space linear. Genal area broader than eye, GW: $\mathrm{EW}=0.9$ : 0.8, surface sculptured as in female. Mesosoma: Pronotum weakly tessellate with minute PP, subapical margin hidden by vertex. Mesoscutum densely tessellate marginally, weakly tessellate medially, shiny with small PP, IS = 1-2. Scutellum weakly tessellate anteriorly with denser PP. Propodeal enclosure weakly rugulate basally, broadly tessellate apically; dorsal face of propodeum strongly roughened. Mesepisternum as in female. Vein 1st $m-c u$ meeting second submarginal cell at middle of cell. Metasoma: Metasomal terga smooth and shiny; tergum 1 with minute PP, IS $=2-4$ or more medially; terga $2-4$ with basal areas with minute PP, IS = 1-2, PP weaker at apical areas; posterior depressions of terga moderately indicated. Sterna smooth and shiny, scattered minute PP; sternum 6 weakly flat, emarginate apically.

Type material. Holotype female, Tianmushan, Zhejiang Province, China, 12. v. 1937 (O. Piel); Paratypes: [CHINA] Zhejiang Province: Tianmushan: 1 female, 12. v. 1937; 1 female, 15. iv. 1936; 1 female, 17 . v. 1936 (O. Piel); Mokanshan: 3 females, 18-25. v. 1936 (O. Piel), 1 male, 25. iv. 1936. Shanghai: 1 female, 10. v. 1918; 1 female, 18. v. 1918 (O. Piel); 1 female, 21. iv. 1930; 1 female, 14. v. 1930 (O. Piel). Shandong Province: 2 females, Qixia, 3. vi. 1967 (J-l. Mao). Sichuan Province: Emeishan: 1 female and 1 male, 14. v. 1964, 2 females, 21. v. 1964 (Y-r. Wu). Liaoning Province: 1 female, Kao-lin-tze, 4-10. vii. 1939 (M. Volkoff).

Remarks. This species is similar to Andrena emeishanica n. sp. in having the brown hairs on head and the large process of labrum, but female can be sepa- rated from that of emeishanica by the pronotum with subapical margin notched medially, the first flagellar segment equals to the next two segments together, and the larger punctures on the metasomal terga. It is also similar to A. watase $i$ Cockerell, but watase $i$ has brown hairs on the mesoscutum and scutellum, the narrowed apex of labral process and the weak and sparser punctures on apical areas of the metasomal terga.

Distribution. China (Shanghai, Zhejiang, Shandong, Sichuan, Liaoning Provs.).

Floral association. Not available.

\section{9-1. Andrena (Melandrena) sibirica Morawitz}

Andrena sibirica Morawitz, 1888, Hor. Soc. Ent. Ross., 22: 239-241 [female, M. Siberia].

Andrena (Melandrena) sibirica: Osytshnjuk, 1995, Key Insects Russian Far East, 4 (1): 504 [female, in key].

Andrena (Zonandrena) sibirica: Gusenleitner and Schwarz, 2001, Entomofauna, 22: 169; Gusenleitner and Schwarz, Entomofauna, Suppl., 12: 694.

Andrena (Gymnandrena) okabei Hirashima, 1957, Mushi, 30: 64-65 [female, China]. New synonymy

Andrena (Melandrena) okabei: Gusenleitner and Schwarz, Entomofauna, Suppl., 12: 552.

Male (new to science). BL 10.0-11.5 mm, WL 9.0-9.5 $\mathrm{mm}(\mathrm{n}=4)$.

Color: Flagellum chocolate beneath except at base; mandible with apical third or more reddened; wing membranes dusky; veins and pterostigma yellowish brown; tibial spurs yellow; posterior depressions of metasomal terga reddish brown.

Pubescence: Hairs on head dense; those on clypeus 300-500 $\mu$, dull white; those on antennal area and genal area dull white; those on vertex 500-700 $\mu$, fulvous. Hairs on thorax dense, dull yellow; those on mesoscutum 300-600 $\mu$, scattered short brown hairs medially; those on scutellum about $700 \mu$; those on mesepisternum and propodeum long, whitish. Hairs on metasomal terga short; those on terga 1-2 white; those on terga 3-5 brown; terga 2-4 with interrupted white hair bands; sterna 2-5 with long, ill-formed white subapical fimbriae.

Structure: Head: HL/HW = 0.77. HW: MsW: MtW = 3.4: 2.8: 3.2. Vertex weakly tessellate with fine small PP, IS $=0.5-1$. OOD: POD: $\mathrm{OCD}=0.7: 0.4: 0.4$. FL1 $>$ FL2, FL2 < FL3, FL2 as broad as long. Eyes with inner margins paralleled. Supraclypeal area coarsely punctate. Face above antennal fossae roughened by coarse interrugal PP. Facial quadrangle broader than long (about 2.2: 2.0). Clypeus strongly convex, weakly tessellate, surface crowdedly punctate with rounded PPø20-40 $\mu$, IS $<0.5$, not roughened, $\mathrm{CPL}=1.0 \mathrm{~mm}$. Process of labrum truncate, weakly emarginate apically, surface smooth and shiny. Labrum apical to process with median cristae. Mandibles decussate. Lower paraocular area smooth and shiny with dense PP, but sparser than in clypeus. Malar space about one-sixth as long as basal mandibular width. Genal area as broad as eye, GW: EW 
= 0.9: 0.9, surface broadly smooth and shiny, sparse PP, IS $=3$ posteriorly, fine minute $\mathrm{PP}$, IS $=1$ near eye. Mesosoma: Pronotum reticularly shagreened with sparse small PP. Mesoscutum finely tessellate marginally, smooth and shiny medially with fine PPø15-20 $\mu$, IS $=0.5-1$. Scutellum sculptured as in mesoscutum, but PP denser. Propodeal enclosure irregularly rugulose mostly, narrowly tessellate apically; dorsal face of propodeum roughened by irregular rugulae. Mesepisternum weakly tessellate, shiny with fine small PP, IS $=1$. Vein 1st $m-c u$ meeting second submarginal cell before middle of cell. Metasoma: Metasomal terga smooth and shiny; terga 1-4 minutely punctate mediobasally with PP, IS = 1-3, distinctly on tergum 2 , smaller and weak $\mathrm{PP}$ at apical areas; posterior depressions of terga moderately indicated. Sterna $2-5$ smooth and shiny with scattered sparse, weak minute PP; sternum 6 flat, not reflected.

Specimens examined. Holotype female of Andrena okabei (Kyushu University), Linkou, Jilin Province, China, 9. viii. 1938 (K. Okabe). Other material: [CHINA] Beijing: 1 female, Baihuashan, 18. viii. 1973 (Y-c. Liu). Hebei Province: Xiaowutaishan, $1200 \mathrm{~m}: 1$ male, 15. viii. 1964; 1 female, 21. viii. 1964; 3 females, 25. viii. 1964 (C-g. Wang); 1 male, 10. viii. 1964; 1 female, 21. viii. 1964; 1 female, 25. viii. 1964; 1 male, 28. viii. 1964; 1 female, 29. viii. 1964 (Y-h. Han). Inner Mongolia Autn. Region: 1 female, Ximeng, 7. viii. 1981 (J-p. Liu). [MONGOLIA] 1 female, Bulgan aimak, $11 \mathrm{~km}$ W. von Somon Bajannauur, 1,000 m, 17. viii. 1968 (Z. Kaszab); 1 male, Cojbalsan aimak, $15 \mathrm{~km} \mathrm{~N}$ von Somon Galuut, 850 m, 17. viii. 1965 (Z. Kaszab).

Distribution. China (Beijing, Hebei, Inner Mongolia, Jilin Provs.). Mongolia (new record, Bulgan, Cojbalsan), Russia (Far East, M. Siberia).

Floral association. Fagopyrum esculentum.

Notes. Mr. Erwin Scheuchl of Germany suggested that the male genitalia of Andrena okabei sapporensis Hirashima is very similar to that of Andrena sibirica Morawitz, during Tadauchi's stay in Linz in 2008. Therefore Tadauchi examined the type specimen of Andrena sibirica Morawitz of St. Petersburg and compared sibirica with the holotypes of okabei Hirashima and okabei sapporensis Hirashima and found they are identical.

\section{9-2. Andrena (Melandrena) sibirica sapporensis Hirashima, new status}

Andrena (Gymnandrena) okabei sapporensis Hirashima, 1957, Mushi, 30: 65 [female, Japan]; Hirashima, 1964, J. Fac. Agr., Kyushu Univ., 13: 63-64; Hirashima, 1966, J. Fac. Agr., Kyushu Univ., 14: 107 [female, in key]; Hirashima and Tadauchi, 1979, Esakia, (14): 135-139 [male].

Specimen examined. Holotype female (Kyushu Univ.), Johzankei, near Sapporo, Hokkaido, Japan, 10. ix. 1916 (S. Matsumura).

Distribution. Japan (Hokkaido, Honshu, Kyushu).

Floral association. Not available.

\section{Andrena (Melandrena) koreana Hirashima}

Andrena (Gymnandrena) koreana Hirashima, 1952, Mushi, 24: 29-30 [female, Korea]; Hirashima, 1957, Mushi, 30: 64; Kim, 1970, Ill. Encycl. Faun. and Flor. Korea, 11: 659 [Korea]; Kim et al., 1990, Ent. Res. Bull., 16: 3 [in list, Korea].

Andrena (Melandrena) koreana: Tadauchi et al., 1997, Esakia, (37): 192-194 [male, Korea]; Gusenleitner and Schwarz, Entomofauna, Suppl., 12: 394-395.

Specimens examined. Holotype female (Kyushu Univ.), Koryo, Korea, 9. v. 1937 (T. Kasanagi). Other material: [CHINA] Heilongjiang Province: 5 females and 1 male, Jingpo Lake, Mudanjiang, 23-26. v. 1993 (O. Tadauchi); Jilin Province: 2 males, Baihe, Changbaishan, 1. vi. 1993 (O. Tadauchi).

Distribution. China (new record, Heilongjiang, Jilin Provs.); Korea (southern and central Korea).

Floral association. China: Taraxacum sp.

\section{Andrena (Melandrena) edashigei Hirashima}

Andrena (Gymnandrena) edashigei Hirashima, 1960, Mushi, 33: 58 [female and male, Japan]; Hirashima, 1964, J. Fac. Agr., Kyushu Univ., 13: 64-66; Hirashima, 1966, J. Fac. Agr., Kyushu Univ., 14: 107, 119 [female and male, in key].

Andrena (Melandrena) edasshigei: Gusenleitner and Schwarz, Entomofauna, Suppl., 12: 242-243.

Specimens examined. Holotype male and allotype female (Kyushu Univ.), Sutaru-toge, Amami-Oshima Is., Kagoshima Pref., Japan, 23. iii. 1954 (T. Edashige).

Distribution. Japan (Amami-Oshima, Okinoerabu Is., Okushima Is.).

Floral association. Not available.

\section{Andrena (Melandrena) gilva n. sp.}

(Fig 5: A-E, Fig. 6: A-E)

Female. BL 12.0-12.7 mm, WL 9.5-9.7 mm ( $\mathrm{n}=7)$.

Color: Flagellum dark reddish brown beneath; mandible with apical half or less reddened; wing membranes subhyaline, yellowish; veins and pterostigma reddish yellow; tibial spurs yellow; posterior depressions of metasomal terga reddened.

Pubescence: Hairs on head moderately dense, yellow but light brown on vertex; those on clypeus 300-400 $\mu$; those on vertex 300-600 $\mu$; facial fovea brown above, whitish below. Hairs on thorax dense, uniformly yellow; those on mesoscutum 400-500 $\mu$; those on scutellum 300-600 $\mu$; those on mesepisternum 600-800 $\mu$, black above, brown below; propodeal corbicula developed with incomplete anterior fringes, with internal simple hairs; trochanteral floccus imperfect; femoral floccus dense; tibial scopal hairs long, simple, yellowish brown. Hairs on metasomal terga rather short, yellow to brown; terga 2-4 with distinct yellowish white hair bands; caudal fimbria brown; sterna $2-5$ with complete yellow subapical fimbriae. 
Structure: Head: HL/HW = 0.79. HW: MsW: MtW = 3.5: 3.5: 4.0. Vertex shagreened by reticular tessellation. OOD: OCD: $\mathrm{POD}=0.7: 0.4: 0.3 . \quad \mathrm{FL} 1=\mathrm{FL} 2+3, \mathrm{FL} 2$ = FL3 as broad as long, intermediate segments about as long as broad. Eyes with inner margins paralleled. Facial fovea exceeding below a line at lower margin of antennal fossae, separated from eye by narrow punctate space, FVL $=1.4 \mathrm{~mm}, \mathrm{FVW}=0.5 \mathrm{~mm}$. Supraclypeal area dulled by weak rugulae. Face above antennal fossae with dense longitudinal rugulae and weak interrugal PP. Facial quadrangle broader than long (about 2.4: 2.2). Clypeus slightly convex, surface densely and reticularly punctate with $\mathrm{PP} \varnothing 20 \mu$, feebly shiny, CPL = $1.1 \mathrm{~mm}$. Process of labrum small, trapezoidal, entire or weakly emarginate apically, surface with transverse rugulae. Labrum apical to process sulcate, without median

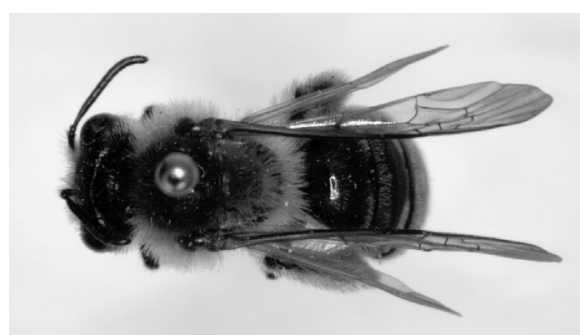

A

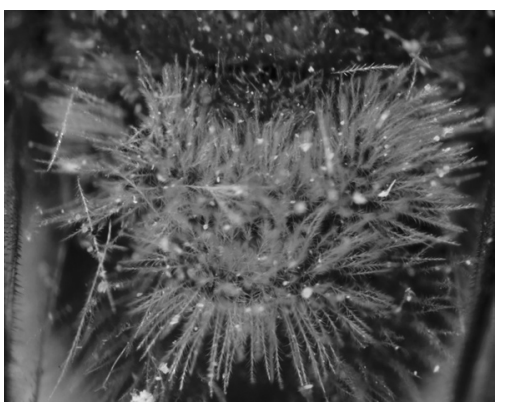

D

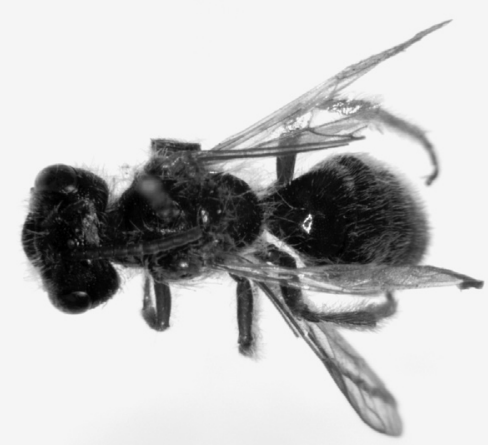

A

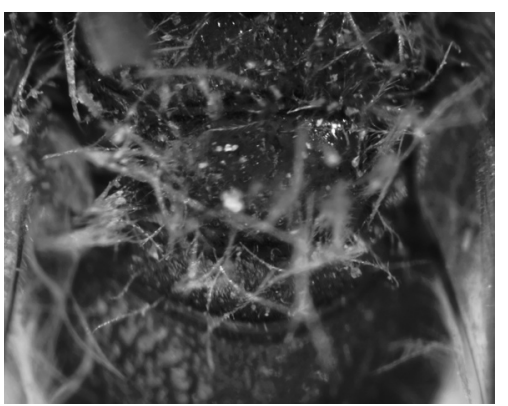

D

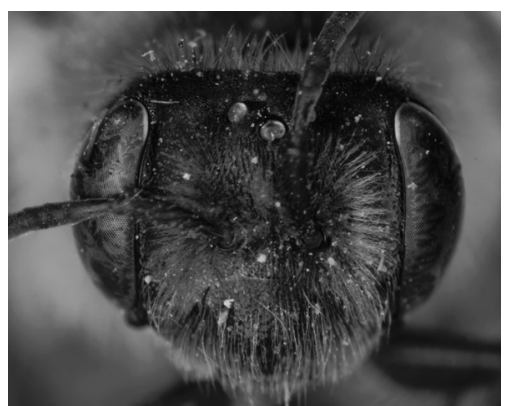

B

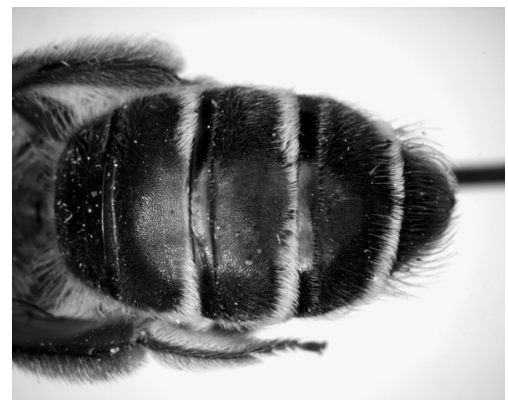

$\mathbf{E}$

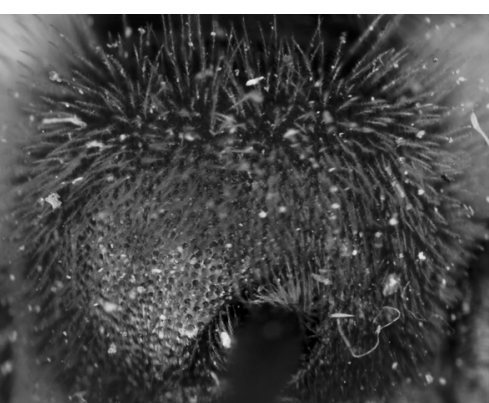

C

Fig. 5. A-E. Andrena (Melandrena) gilva n. sp., female. A general habitus in dorsal view; B: head in frontal view; C: mesoscutum; D: propodeum; E: metasomal terga.

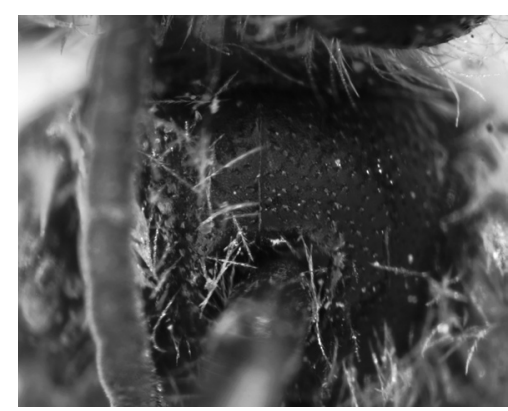

C

Fig. 6. A-E. Andrena (Melandrena) gilva n. sp., male. A: general habitus in dorsal view; B: head in frontal view; C: mesoscutum; D: propodeum; E: metasomal terga.

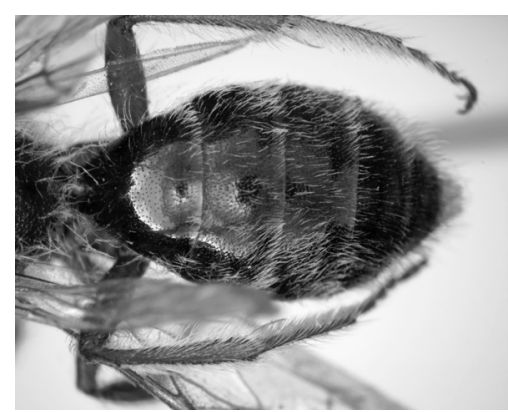

$\mathbf{E}$

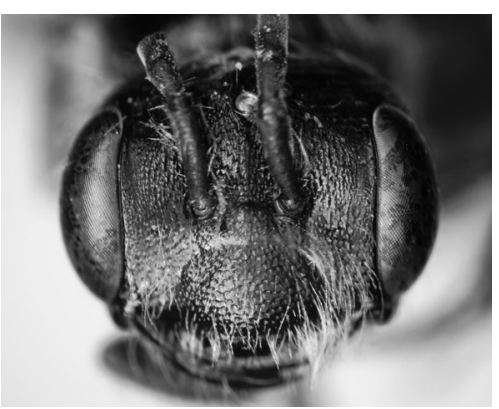

B 
cristae. Lower paraocular area as in clypeus, but PP smaller. Malar space nearly linear, broadened posteriorly. Genal area as broad as eye, GW: EW = 0.9: 0.9, surface weakly tessellate, shiny, sparsely punctate posteriorly. Mesosoma: Pronotum weakly tessellate, surface shiny with dense minute PP posteriorly. Mesoscutum coarsely punctate with PРø20 $\mu$, IS $<0.5$, feebly shiny. Scutellum as in mesoscutum. Propodeal enclosure narrow, finely rugulose basally, granulate apically; dorsal face of propodeum rugoso-punctate, roughened. Mesepisternum finely tessellate, shagreened medially. Vein 1 st $m-c u$ meeting second submarginal cell at middle of cell. Metasoma: Metasomal terga densely punctate, narrowly shiny. Tergum 1 with PPø10-20 $\mu$, IS < 1; terga 2-4 with PPø15 $\mu$, IS $<0.5$ at basal areas, smaller and close minute PP at apical areas; posterior depressions of terga narrow, not well indicated; pygidial plate V-shaped with rounded apex, internal area flat. Sterna 2-5 weakly tessellate, impunctate basally, densely punctate apically.

Male. BL 9.0 mm, WL $7.7 \mathrm{~mm}(\mathrm{n}=1)$.

Color: Flagellum reddish brown beneath; mandible with apical third reddened; wing membranes subhyaline, yellowish; veins and pterostigma reddish yellow; tibial spurs yellowish; posterior depressions of metasomal terga yellowish brown.

Pubescence: Hairs on head moderately dense, white but those on antennal area and vertex mixed with brown; those on clypeus 400-600 $\mu$; those on vertex 200-500 $\mu$. Hairs on thorax dense, dull white to white; those on mesoscutum and scutellum 300-500 $\mu$, dull white mixed with brown; those on mesepisternum $700 \mu$, white. Hairs on metasomal terga short, dense; terga 1-3 with dull white hairs; terga 4-5 with brown ones; terga 2-4 with interrupted, weak white hair bands; caudal fimbria brown; sterna 2-4 with incomplete sparse whitish subapical fimbriae, sternum 5 with brown hairs.

Structure: Head: HL/HW = 0.76. HW: MsW: MtW = 2.9: 2.5: 2.7. Vertex densely tessellate with fine minute PP. OOD: POD: $\mathrm{OCD}=0.6: 0.4: 0.3$. FL1 about 1.4 times as long as FL2, FL2 as broad as long, following segments longer than broad. Eyes with inner margins paralleled. Supraclypeal area and face above antennal fossae sculptured as in female. Facial quadrangle broader than long (about 2.0: 1.7). Clypeus well convex, surface rugoso-punctate, shagreened, $\mathrm{CPL}=0.8 \mathrm{~mm}$. Process of labrum small as in female, weakly emarginate apically, surface smooth and shiny. Mandibles decussate. Lower paraocular area densely punctate with PPø10$20 \mu$. Malar space nearly linear. Genal area as broad as eye, GW: EW = 0.8: 0.8, surface sculptured as in female. Mesosoma: Pronotum weakly tessellate, surface shiny with fine PP. Mesoscutum weakly tessellate except anteriorly, weakly shiny with minute PP, IS $<1$. Scutellum densely tessellate posteriorly, weakly shiny with close minute PP anteriorly. Propodeum and mesepisternum sculptured as in female. Wing venation as in female. Metasoma: Metasomal terga weakly tessellate, shiny with fine minute PP, IS = 1-2, sparser on tergum 1; posterior depressions of terga narrow, well indicated. Sterna
2-5 weakly tessellate, broadly shiny, impunctate at basal halves, weakly punctate at apical halves.

Type material. Holotype female, Kuling, Jiujiang, Jiangxi Province, China, 4. ix. 1936 (O. Piel). Paratypes: [CHINA] 2 females, the same data as the holotype; 1 female, 1. ix. 1934. Hunan Province: Shanzi County: 2 females and 1 male, Tianpingshan, 1,350 m, 12. viii. 1988 (L-l. Yang). Anhui Province: 1 female, Huangshan, 24. ix. 1995 (Y. Yamagishi).

Remarks. This species is similar to Andrena koreana Hirashima in having the small labral process and abundant yellow hairs on thorax. The female can be separated from that of koreana by the clypeus less roughened, smaller punctures on the mesoscutum and metasomal terga, and the white tibial scopal hairs. The male can be separated from that of koreana by the less convex clypeus, the first flagellar segment longer than second flagellar segment and denser punctures on the metasomal terga.

Distribution. China (Jiangxi, Hunan, Anhui Provs.). Floral association. Not available.

\section{Andrena (Melandrena) anzu Tadauchi et Hirashima}

Andrena (Melandrena) anzu Tadauchi et Hirashima, 1987, Esakia, (25): 135-137 [female, Japan]; Gusenleitner and Schwarz, Entomofauna, Suppl., 12: 89.

Specimen examined. Holotype female (Type No. 2586, Kyushu Univ.), Mori, Koshoku, Nagano Pref., Japan, 21-22. iv. 1963 (Y. Maeta).

Distribution. Japan (central Honshu).

Floral association. Prunus armeniaca.

\section{Andrena (Melandrena) comta Evensmann}

Andrena comta Eversmann, 1852, Bull. Soc. nat. Moscou, 25(2): 12 [female and male, Russia].

Andrena (Melandrena) comta: Gusenleitner and Schwarz, 2000, Entomofauna, 21 (10): 113[species recovery]; Gusenleitner and Schwarz, Entomofauna, Suppl., 12: 197-198.

Andrena orenburgensis Schmiedeknecht, 1883, Apid. Europ., 1: 831-832, new name for Andrena compta Eversmann [nec. Andrena compta Lepeletier, 1841]; Morawitz, 1890, Horae Soc. ent. Ross., 24: 357 [in list, Mongolia]; Yasumatsu, 1941, Peking nat. Hist. Bull., 15: 280 [in list].

Andrena (Glyphandrena) wulungshanensis Yasumatsu, 1935, Insect Jehol, 8: 2, 36 [female, China]; Okabe, 1939, Trans. Kansai ent. Soc., (8): 12 [in list]; Yasumatsu, 1941, Peking nat. Hist. Bull., 15: 282 [in list]; Yasumatsu, 1947, Mushi, 18: 32 [in list].

Andrena (Gymnandrena) wulungshanensis: Hirashima, 1957, Mushi, 30 (10): 65 [male, Japan]; Hirashima, 1964, J. Fac. Agr., Kyushu Univ., 13: 57; Hirashima, 1966, J. Fac. Agr., Kyushu Univ., 14: 107, 118 [female and male, in key]. 
Andrena (Melandrena) orenburgensis: Warncke, 1968, Mem. Est. Mus. Zool. Univ. Coimbra, (307): 78; Osytshnjuk, 1995, Key Insects Russian Far East, 4 (1): 504, 523 [female and male, in key]; Xu and Tadauchi, 1997, J. Fac. Agr., Kyushu Univ., 41: 176-177.

Specimens examined. [CHINA] Inner Mongolia Autn. Region: 1 female, Humeng, 2. viii. 1981 (D-X. Liao); 1 male, Xilinhaote, 25. vii. 1972. Xinjiang Uygur Autn. Region: 1 male, Fuhai, 500 m, 21. vii. 1960 (S-y. Wang); 1 male, Aletai, 800 m, 22. vii. 1960 (F-c. Zhang). Heilongjiang Province: 1 female, Harbin, 8. viii. 1940 (S. Asahina); 1 male, Harbin, 9. viii. 1955; Gansu Province: 3 females and 1 male $(\mathrm{H}-\mathrm{l}, \mathrm{Xu}) ; 3$ females and 1 male (O. Tadauchi), Wuwei city, 11. viii. 1996. [MONGOLIA] Cojbalsan aimak: 4 females, 13-18. viii. 1965; 4 males, 28. vii-17. viii. 1965 (Z. Kaszab); Suchebaator aimak: 5 males, 24. vii-3. viii. 1965 (Z. Kaszab).

Distribution. China (Xinjiang, Gansu, Inner Mongolia, Heilongjiang Provs.); Japan (Honshu); Mongolia (Cojbalsan, Suchebaator); Russia (Far East, European Russia); M. Europe.

Floral association. China: Brassica campestris.

\section{Andrena (Melandrena) emeishanica n. sp.}

(Fig. 7: A-E)

Female. BL $14.5 \mathrm{~mm}$, WL $11.2 \mathrm{~mm}(\mathrm{n}=1)$.

Color: Flagellum reddish beneath; mandible reddened from apex to base; wing membrane moderately brown, veins and pterostigma reddish brown; tibial spurs testaceous; posterior margins of metasomal terga yellow subhyaline.

Pubescence: Hairs on head moderately dense, fulvous to brown; those on clypeus 400-500 $\mu$, naked medially; those on vertex 500-600 $\mu$, dark brown; those on genal area fulvous; facial fovea brown. Hairs on thorax dense, yellow; those on mesoscutum and scutellum 300-600 $\mu$, those on mesepisternum paler; propodeal corbicula developed with incomplete anterior fringes, with internal long, dense simple hairs; trochanteral floccus imperfect, dull white; femoral floccus dense; tibial scopal hairs long, simple, brown; basitarsus with brown hairs. Hairs on metasomal terga short, brown except on tergum 1 moderately long, white laterally and apically; terga $2-4$ with short, white hair bands, interrupted on tergum 2; caudal fimbria blackish; sterna 2-5 with long, sparse brown subapical fimbriae.

Structure: Head: HL/HW = 0.81. HW: MsW: MtW = 4.0: 4.0: 4.5. Vertex finely tessellate and weakly punctate. OOD: OCD: $\mathrm{POD}=0.8:$ 0.5: 0.4. FL1 > FL2+3, FL2 = FL3 longer than broad. Eyes with inner margins paralleled. Facial fovea extending to below a line at lower margin of antennal fossae, separated from inner margin of eye by broad punctate space, $\mathrm{FVL}=1.5 \mathrm{~mm}$, $\mathrm{FVW}=0.5 \mathrm{~mm}$. Supraclypeal area dulled by irregular rugulae. Face above antennal fossae with fine rugulae and PP, surface shagreening. Facial quadrangle broader than long (about 3.0: 2.6). Clypeus well convex, sparsely tessellate-punctate laterally, smooth and shiny medially, surface with $\mathrm{PP} \varnothing 40 \mu$, IS $=0.5-1$ laterally, sparser toward median area with PPø20-40 $\mu$ medioapically, irregular in distribution; $\mathrm{CPL}=1.3 \mathrm{~mm}$. Process of labrum large, trapezoidal, emarginate apically, surface with rugulae at base. Labrum apical to process transversely sulcate, without crista. Lower paraocular area weakly tessellate, weakly punctate with PPø20 $\mu$, IS = 1. Malar space about one-fifth as long as basal mandibular width. Genal area broader than eye, GW: EW = 1.2: 0.8 , surface weakly tessellate, scattered obscure minute $\mathrm{PP}$ posteriorly, fine PP, IS $=0.5-1$ near eye. Mesosoma: Pronotum finely tessellate with obscure PP. Mesoscutum densely tessellate anteriorly, weakly tessellate at the remainder, surface feebly shiny with PPø20 $\mu$, IS = 0.5-1. Scutellum densely tessellate posteriorly, smooth and shiny anteriorly with PPø20 $\mu$, IS $=0.5-2$. Propodeal enclosure strongly rugose basally with distinct plicate rugulae, weakly rugulose apically; dorsal face of propodeum shagreened by coarse tessellation. Mesepisternum strongly roughened medially by coarse punctation. Vein 1st $m-c u$ meeting second submarginal cell beyond middle of cell. Metasoma: Metasomal tergum 1 smooth and shiny with crowded PPø15 $\mu$, IS $=0.5-1$; terga $2-4$ similarly punctate with close minute PP, IS $<0.5$; posterior depressions of terga indistinct; pygidial plate U-shaped without raised internal area. Sterna 2-5 weakly tessellate apically with fine PP, smooth and shiny basally without PP.

Male. Unknown.

Type material. Holotype female, Emeishan, 550750 m, Sichuan Province, China, 10. v. 1957 (Y-c. Lu).

Remarks. This species is a relative to preceding species Andrena gilva n. sp. in having the strongly convex clypeus, the rather closely punctate mesoscutum and metasomal terga. The female can be separated from that of gilva by the process of labrum larger and deeply emarginate, the first flagellar segment longer than the flagellar segment 2 plus 3 , the broadly rugulate propodeal enclosure and the larger size in length.

Distribution. China (Sichuan Prov.).

Floral association. Not available.

\section{Andrena (Melandrena) sasakii Cockerell}

Andrena sasakii Cockerell, 1913, Ann. Mag. nat. Hist., (8)11: 189 [male, Japan]; Yasumatsu, 1941, Peking nat. Hist. Bull., 15: 280 [in list]; Uéda, 1954, Trans. Shikoku ent. Soc., 4: 50 [in list].

Andrena (?Gymnandrena) sasakii: Hirashima, 1964, J. Fac. Agr., Kyushu Univ., 13: 66-69 [female and male, redescription].

Andrena (Gymnandrena) sasakii: Tadauchi, 1989, check list Jap. Insects, 683 [in list]; Kim et al., 1990, Ent. Res. Bull., 16: 4 [Korea]; Xu and Tadauchi, 1996, Bull. biogeogr. Soc. Jap., 51: 1-2.

Anthrena consimilis Alfken, 1900, Ent. Nachr., 26: 177 [female, Japan].

Andrena consimilis (nec consimilis Smith, 1847): Cockerell, 1913, Ann. Mag. nat. Hist., (8)11: 190 [female, in key, Japan]; Yano, 1932, Icon. Ins. Jap., 


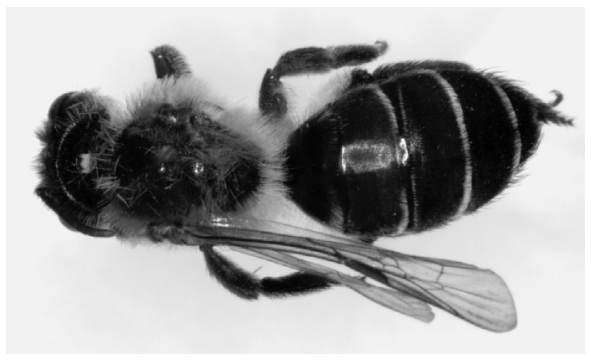

A

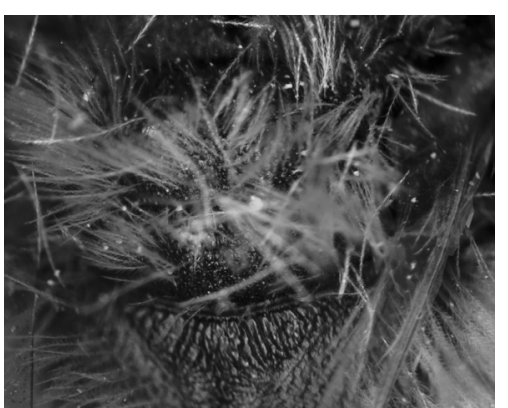

D

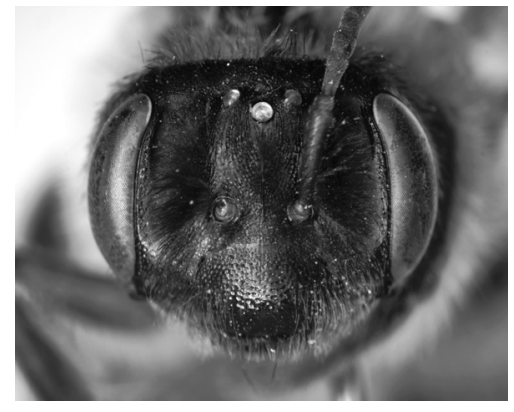

B

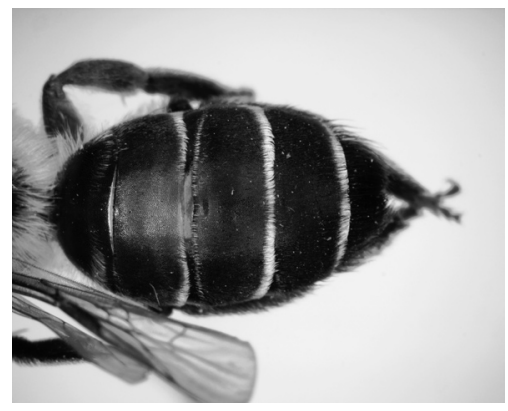

$\mathbf{E}$

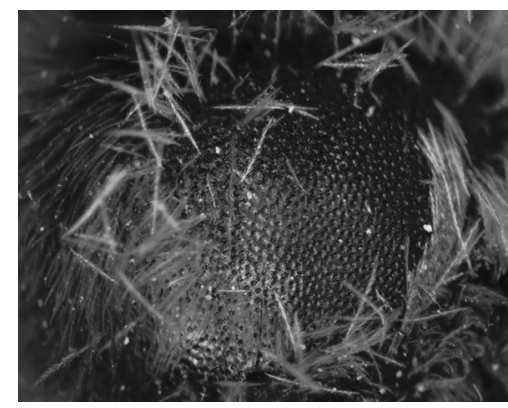

C

Fig. 7. A-E. Andrena (Melandrena) emeishanica n. sp., female. A: general habitus in dorsal view; B: head in frontal view; C: mesoscutum; D: propodeum; E: metasomal terga.

272 [female, Japan]; Kato, 1938, Insect-fauna Musashino, 152 [in list]; Yasumatsu, 1941, Peking nat. Hist. Bull., 15: 280 [in list].

Andrena plesia Viereck, 1918, Proc. biol. Soc. Washington, 31: 59, new name for Andrena consimilis Alfken.

Andrena alopex Cockerell, 1917, Entomologist, 50: 86 [female, Japan].

Andrena (Melandrena) sasakii: Gusenleitner and Schwarz, Entomofauna, Suppl., 12: 661-662.

Specimens examined. [CHINA] Shanghai: 1 male, 19. iv. 1935 (O. Piel); 1 male, 22. iv. 1933 (A. Savio); 1 male, 13. iv. 1955 (K-r. Huang). Jiangsu Province: 1 female, Zhenjiang, 1. iv. 1918; 1 female, Zhenjiang, 1. iv. 1919 (O. Piel); 2 females, Chemo, 30. iv.-3. v. 1935 (O. Piel). Zhejiang Province: 2 females, Hangzhou, 16. ix. 1932; 6 females, Tianmushan, 20. iv. 1996 (O. Tadauchi and $\mathrm{H}-\mathrm{l}$. Xu). Shandong Province: 1 male, Yantai, 16. iv. 1961 (Y-l. Chen). Shannxi Province: 1 female, Northwest Agricultural University (Yangling), 21. iv. 1955 (S-j. Ma).

Distribution. China (new record, Shanghai, Zhejiang, Shandong, Shannxi Provs.); Japan (Honshu, Sado Is., Shikoku, Kyushu, Tsushima Is., Taneagashima Is.); Korea (central Korea).

Floral association. Prunus japonica.

\section{Andrena (Melandrena) vaga Panzer}

Andrena vaga Panzer, 1799, Faun. Insect. German., 64: 18 [female, Austria].

Andrena ovina Klug, 1810, Magazin Ges, Naturf. Fr. Berl., 4: 266-270 [female and male, Germany].

Andrena nitidiventris Blanchard, 1840, Hist. Nat. Anim. Artic.: 414 [female, M. France].

Andrena nitidiventris Dufour, 1841 (nec Andrena nitidiventris Blanchard, 1840), Mem. Acad. Sci,
Inst. Fr., 7: 456 [N. France].

Andrena atricula Bischoff, 1922, Dt. Ent. Z., 1922: 428-429 [male, N. Germany].

Andrena (Melandrena) vaga: Warncke, 1967, Eos, Madr., 43: 82; Osytshnjuk, 1977, Fauna Ukraini, 12 (5): 207-209; Osytshnjuk, 1995, Key Insects Russian Far East, 4 (1): 504 [female, in key]; Gusenleitner and Schwarz, 2002, Entomofauna, Suupl., 12: 798-800; Tadauchi, 2008, Esakia, (48): 14.

Other synonymies: See Gusenleitner and Schwarz (2002).

Only cited from Osytshnjuk (1995).

Distribution. Russian Far East, Kazakhstan, Kyrgyzstan, Kaukasus, European Russia, M. and N. Europe.

\section{ACKNOWLEDGEMENTS}

We wish to express our cordial thanks to Emeritus Y. Hirashima of Kyushu University and Prof. Wu Yan-ru of Institute of Zoology, Academia Sinica for their constant guidance and various help. We also thank Mr. E. Scheuchl of Velden, Germany for his valuable information on Andrena sibirica. We are indebted to Ms. C. Hirosawa for preparing photos of this manuscript. This work was supported by a Grant-in-Aid for Scientific Research of an International Scientific Research Program from the Ministry of Education, Science, Sports and Culture, Japan (No. 07041144) and (B) (2) from Japan Society for the Promotion of Science (No. 14405025) (Both of head investigators: O. Tadauchi). This is Result from the China-Japan Co-operative Study on "Studies on Systematics, Evolution and Biogeography of Asian Andrena (Hym., Apoidea, Andrenidae)" No. 20. This is a Contribution from the Entomological 
Laboratory, Faculty of Agriculture, Kyushu University, Fukuoka (Ser. 6, No. 61).

\section{REFERENCES}

Bouseman, J. K. and W. E., LaBerge 1978 A revision of the bee of the genus Andrena of the western hemisphere. Part IX. Subgenus Melandrena. Trans. Amer. Ent. Soc., 104(3-4): 275-389

Gusenleitner, F. and M. Schwarz 2000 Nomenklatorische Aktualisierungen in der Bienengattung Andrena sowie Beschreibung einer neuen Art (Hymenoptera: Apidae: Andreninae). Entomofauna, 21(10): 105-116

Gusenleitner, F. and M. Schwarz 2001 Zur Morphologie verschiedener von F. Morawitz beschriebener Andrena-Arten (Hymenoptera: Apidae: Andrenidae). Entomofauna, 22(7): 93-196

Gusenleitner, F. and M. Schwarz 2002 Weltweite Checkliste der Bienengattung Andrena mit Bemerkungen und Ergänzungen zu paläarktischen Arten (Hymenoptera, Apidae, Andreninae, Andrena). Entomofauna, 12(Suppl.): 1-1280

Hedicke, H. 1933 Beitrage zur Systematik der Gattung Andrena F, (Hym. Apid.). Mitt. Zool. Mus. Berlin, 19: 199-220

Hirashima, Y. 1964 Systematic and biological studies of the family Andrenidae of Japan (Hymenoptera, Apoidea). Part 2. Systematics, 3. J. Fac. Agr., Kyushu Univ., 13(1): 40-69

Kim, C.-w. 1970 Illustrated Fauna and Flora of Korea, 11(3), 835 pp., Seoul

Kim, M-l., C-w. Kim and C-s. Kim 1990 Systematic study of genus Andrena (Andrenidae; Hymenoptera) from Korea II.
Subgenus Gymnandrena. Ent. Res. Bull., 16:1-10

LaBerge, W. E. 1964 Prodromus of American bees of the genus Andrena (Hymenoptera, Apoidea). Bull. Univ. Nebraska St. Mus., 4: 279-316

Lanham, U. 1949 A subgeneric classification of the New World bees of the genus Andrena. Univ. California Publ. Ent., 8 : 183-237

Osytshnjuk, A. Z. 1995 Andrena. In "Key to the insects of Russian Far East in six volumes. vol. 4, Neuropteroidea, Mecoptera, Hymenoptera, Part 1. 606pp, St. Petersburg: Nauka, pp. 489-527 (In Russian)

Tadauchi, O. 2008 The genus Andrena from Kazakhstan and Kyrgyzstan (Hymenoptera, Andrenidae) (2). Esakia, (48) $1-18$

Tadauchi, O., H-l. Xu and J-c. Paik 1997 The family Andrenidae of Korea (Hymenoptera, Apoidea) II. Esakia, (37): 187-202

Warncke, K. 1968 Die Untergattungen der westpaläarktischen Bienen-gattung Andrena F. Mem. Est. Mus. Zool. Univ. Coimbra, (307): 1-111

Wu, Y-r. 1965 Economic Insects Fauna of China IX, Hymenoptera, Apoidea. Sci. Publ., Beijing, 83 pp, 7 pls

Wu, Y-r. 1982 Hymenoptera, Apoidea. In "Insects of Xizang", Vol. 2, pp. 379-426, Science Press, Beijing, 508 pp (In Chinese with English summary)

Yasumatsu, K. 1935 Reports of the first scientific expedition to Manchoukuo. Insects of Jehol, fasc. 8, Apoidea

Yasumatsu, K. 1941 A list of the Far Eastern species of the genus Andrena (Hym., Apoidea). Peking nat. Hist. Bull. 15: 273-284 